\title{
Monde moderne et/vs monde classique - compatibles?
}

\author{
Jean Martin
}

Dr med., membre de la rédaction

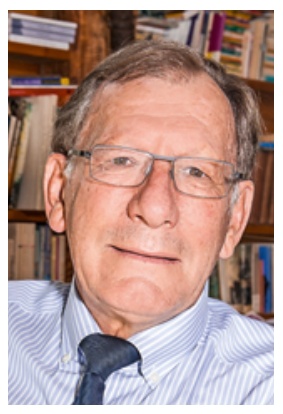

Bientôt la fin de l'année, moment double de bilan et de regard vers l'avant. Je suis frappé par des ouvrages récents de philosophes solides très critiques de l'évolution que nous vivons. Le Français Eric Sadin (1973) s'élève contre la «sili-colonisation» du monde. Il est préoccupé par notre dérive vers une société mettant tellement l'accent sur la mesure [1]. NB: pas du tout la mesure comme pondération ou bon sens, mais comme quantification obsessionnelle, dans la vie des gens et des sociétés. La poussée vers le quantified self en est une illustration. Dans la foulée, il veut un renouveau de l'humanisme, mais voit rouge si on parle d'humanités digitales! A la française, il appelle, dans un sens qualitatif, à un retour à la réflexion et aux valeurs classiques des mouvements issus de la société civile devraient promouvoir cette réorientation. Mais si on constate aujourd'hui, heureusement, des démarches vers des modes de vie et de production alternatifs, je n'y vois guère de lame de fond anti-digitalisation. En médecine par exemple, peu probable que beaucoup refusent les investigations de plus en plus fines qu'elle permet.

Le penseur majeur qu'est Peter Sloterdijk (1947), philosophe allemand enseignant à Karlsruhe, oppose dans «Après nous le déluge» [2] classicisme et modernité, débattant du déséquilibre d'un monde affranchi du passé. «Moderniser, c'est aller toujours plus loin dans la direction du déracinement, du déni des origines, et dans la direction d'une vie sans descendance." Aussi: «Le monde classique était structuré par cette différence essentielle: la mortalité des individus et la permanence des cultures. C'était son ontologie. La modernité introduit, dans les traces du darwinisme, l'idée que même les civilisations peuvent disparaitre» comme l'a dit Paul Valéry il y un siècle.

Sloterdijk s'intéresse aux liens entre générations: «Au fond, il n'y a jamais eu une véritable continuité entre les parents et les enfants. Mais le monde ancien a essayé, avec un succès considérable, de faire en sorte que l'apparence de la continuité l'emporte sur la discontinuité. A partir de la Renaissance, les choses ont changé, avec un nouveau cycle où les innovateurs entrent en scène pour pousser les savoirs en avant. La modernisation ouvre des écarts entre les générations.»
Une de ses opinions m'interpelle, à l'encontre de ma conviction que le métissage est tonique, enrichissant: «Dans la réflexion moderne, il n'y a rien de plus chic que la bâtardise, qu'on appelle avec fierté le métissage. Chez les modernes, il y a la volonté d'élargir les frontières du normal» (on ne sera pas étonné qu'il ne pense rien de bien du transhumanisme). «Ce qui fait que la modernité, c'est la tolérance bien sûr, mais en même temps c'est une civilisation qui risque de se débarrasser d'elle-même.» Well... je reste (J.M.) de l'avis que la capacité de tolérance est importante et qu'il s'agit de gérer au mieux les poussées vers le mélange et la mixité (qu'il n'est pas imaginable d'interdire ou annihiler) - en refusant par principe le métissage on glisse vers des régimes autoritaires.

Sloterdijk sur que nous vivons: "La maitrise d'un véhicule dépend de l'existence d'un moyen d'arrêter le mouvement. Notre civilisation ne connaît que la moitié de cette maitrise [l'accélération], et le processus est incontrôlable. Voyage dans un train sans pilote et sans freins, on ne sait même pas si les rails ont été posés.» Que penser quand un esprit de cette qualité affirme que «la plus grande des superstitions, c'est la conviction de marcher à la tête du progrès»! On songe aussi aux débats sur l'intelligence artificielle quand, en réponse aux craintes exprimées, il est affirmé que jamais au grand jamais des dispositifs non-humains ne décideront à notre place, que nous pourrons toujours corriger assez tôt. Cependant: dans un monde hyperconnecté, avec des "clouds" immenses de données qu'utiliseront ou interpréteront des machines, quelle assurance que l'humain gardera la maitrise ultime? Je m'apprête à passer les Fêtes en famille, avec enfants et petits-enfants, en jouissant intensément de ces interactions. Mais je reste songeur. Heureusement, Sloterdijk affirme qu'il faut être un optimiste imperturbable. Mais il ne dit pas comment.

Bonne année à tou(te)s!

\section{Références}

1 Sadin E. La silicolonisation du monde. Paris: Ed. L'Echappée, 2016. Aussi: Entretien sur la radio RTS «La Première», $7 \mathrm{~h} 35$ - 8h, $1^{\text {er }}$ novembre 2016

2 Sloterdijk P. Après nous le déluge. Paris: Payot, 2016. Aussi: Interview à ce propos dans Le Figaro (Paris), 23 octobre 2016, d'où sont tirées les citations. 